\title{
Ricordo di Renato Traini \\ (Mezzoldo, 16 agosto 1923-Roma, 27 giugno 2014)
}

\author{
Roberta Denaro \\ Università di Napoli “L’Orientale” \\ rdenaro@unior.it
}

È strano, a distanza di vent'anni, guardare alle lezioni di Renato Traini, una delle esperienze che più terrorizzava gli studenti di arabo alla Sapienza, come a un privilegio di cui essere grati alla sorte. Di quelle lezioni, ricordo la voce sottile del professore (incisa su nastri ormai inservibili) leggere e commentare in aula centinaia di pagine, esempi tangibili della bellezza, e delle asperità, di una grande tradizione letteraria che Traini ha presentato a generazioni di studenti, con una conoscenza della lingua sorprendente per finezza e rigore, non senza, forse, una certa ritrosia a condividere con noi passioni intellettuali di cui ci considerava incapaci.

La sensazione di privilegio, che condivido con altri che meglio di me potrebbero raccontare Renato Traini per averlo più assiduamente frequentato, è resa più acuta dalla consapevolezza di avere avuto di fronte uno dei massimi, nonché ultimi, esponenti di una tradizione che ha annoverato studiosi come Carlo Alfonso Nallino (1872-1938), Giorgio Levi Della Vida (1886-1967), Francesco Gabrieli (1904-1996) e molti altri che nel Novecento hanno costruito gli studi arabi e islamici in Italia.

Secondo un profilo da orientalista "classico", Renato Traini ebbe una formazione ampia che comprendeva ovviamente il greco e il latino (che insegnò per circa dieci anni nella scuola superiore) e non fu ristretta all'arabo: si laureò in ebraico all'Università di Pavia nel 1946, prima di giungere all'arabo, studiato alla Statale di Milano e a Parigi, e al siriaco di cui seguì i corsi al Pontificio Istituto Biblico.

A partire dal $195^{8}$ curò lo spoglio delle stampa araba (sezione "Arabia Saudiana") per Oriente Moderno, all'epoca rivista mensile. Pochi anni dopo, quasi ripercorrendo le orme di Francesco Gabrieli, che di Traini fu mentore e amico, fu chiamato come conservatore della Sezione orientale della Biblioteca dell'Accademia dei Lincei (dal 1962 al 1979) e infine come professore di Lingua 
araba alla Sapienza romana (incarico che coprì dal 1979, dopo la prematura scomparsa di Paolo Minganti, al 1993).

Non è però qui mia intenzione tracciare un quadro completo della carriera scientifica e professionale di Renato Traini, cosa che è già stata, peraltro ottimamente, fatta, ${ }^{1}$ quanto piuttosto delineare in breve i tratti salienti della sua biografia intellettuale e soprattutto rendere omaggio a uno studioso che ha rappresentato a livelli altissimi una tradizione scientifica e una visione dell'arabistica che oggi appaiono, per molte ragioni, distanti. I suoi corsi di arabo frequentati da cinque o sei studenti, minoranza sparuta ed "eccentrica" tra quanti sceglievano di studiare lingue negli anni novanta del secolo scorso (che impressione anche solo scriverlo ...), sembrano, infatti, molto lontani se confrontati a quelli affollati di questi anni in cui mutamenti epocali hanno modificato tangibilmente il rapporto della società italiana con il mondo arabo e, di riflesso, anche il contesto e gli obiettivi della didattica universitaria della lingua araba.

E tuttavia, benché l'insegnamento dell'arabo abbia preso direzioni che Renato Traini avrebbe considerato forse lontane dai propri percorsi, è a lui che si deve uno dei più longevi strumenti di studio e di lavoro per chiunque si avvicini a questa lingua, il Vocabolario arabo-italiano (pubblicato dall'Istituto per l'Oriente tra il 1966 e il 1973 e continuamente ristampato). ${ }^{2}$ A questo dizionario che rimane per tutti, semplicemente, "il Traini", è affidata in primo luogo la memoria del suo nome benché l'autore ne abbia firmato, con modestia, solo la prefazione in cui dichiarava la volontà di "colmare una lacuna vivamente sentita dagli arabisti italiani" ${ }^{3}$ non senza chiedere al lettore "qualche indulgenza" per i possibili errori in "questo non lieve lavoro". ${ }^{4}$

1 Una sua esaustiva biografia è stata pubblicata da Carmela Baffioni. "Ricordo di Renato Traini". Atti dell'Accademia nazionale dei Lincei. Rendiconti della classe di scienze morali, storiche e filologiche, s. 9, XXVI (2015), p. 301-315. Renato Traini è stato ricordato in due distinte occasioni anche da Angelo Arioli: "Lessico, codici, passioni yemeniche ... ricordo di Renato Traini". In: Miscellanea Arabica 2013-2014. Ariccia, Aracne Editrice (Nuova Sapienza Orientale, 5), 2014, p. 15-39, e "Ricordo di Renato Traini". RSO, LXXXVIII/1-4 (2015), p. 57-71. Per una rassegna più ampia dell'opera di Renato Traini rimando a questi contributi.

2 Il dizionario, alla cui preparazione Traini lavorò a partire dal $195^{8}$ prima in collaborazione con Maria Nallino (fino alla pagina 240 delle complessive 1763) e poi da solo, uscì anonimo in tre volumi tra il 1966 e il 1973: Vocabolario arabo-italiano. Vol. I (alif-sin). Roma, Istituto per l'Oriente, 1966; Vocabolario arabo-italiano. Vol. II (šin-z̧a $\bar{a}^{3}$. Roma, Istituto per l'Oriente, 1969 e Vocabolario arabo-italiano. Vol. III ( $q \bar{a} f-y \bar{a})$. Roma, Istituto per l'Oriente, 1973.

3 Traini. Vocabolario arabo-italiano. I. viII.

4 Ibid., p. Ix. 
Se il dizionario è il lascito più noto di Renato Traini, è allo studio dei testi manoscritti che egli dedicò gran parte della propria vita professionale, quasi in risposta al "grido di dolore" lanciato alla fine degli anni quaranta del Novecento da Olga Pinto che, facendo un bilancio della situazione dei fondi orientali nelle biblioteche italiane, lamentava la mancanza di un'adeguata opera di descrizione e catalogazione dell'intero patrimonio di tali manoscritti. ${ }^{5}$ A quest'impresa si è a lungo dedicato Renato Traini, adoperandosi come pochi altri a "far conoscere ciò che ancora è quasi o affatto sconosciuto e far conoscere meglio o più compiutamente quello che è rimasto indicato in modo provvisorio e parziale o succinto". ${ }^{6}$ A lui si devono infatti strumenti di orientamento tuttora utilissimi per la conoscenza di questo notevole patrimonio manoscritto ${ }^{7}$ nonché un'attività catalografica che ne ha colmato vuoti importanti e che resta un modello per competenza e rigore metodologico. Tale lavoro paziente e incessante, iniziato nel $1967^{8}$ e conclusosi nel $2011,{ }^{9}$ fu probabilmente quello a lui

5 Pinto, O. "Manoscritti e stampati orientali nelle Biblioteche governative italiane". Rso, xxIV (1949), p. 161-168.

6 Traini, R. "I fondi manoscritti arabi in Italia". In: Gli studi sul Vicino Oriente in Italia dal 1921 al 1970. II. L'Oriente islamico. Roma, Istituto per l'Oriente, 1971, p. 247. Questo saggio resta tuttora un'indagine fondamentale sulla storia e la consistenza dei fondi manoscritti arabi presenti nelle biblioteche italiane. A ripresa e integrazione di questo studio si aggiungono, più recentemente, P. Orsatti, B. Pirone e A. Gallotta, "Italy". In: World Survey of Islamic Manuscripts. II. London, al-Furqān Islamic Heritage Foudation, 1993, p. 67-116, e il bilancio dei lavori di catalogazione sui codici arabi curato da V. Sagaria Rossi "La catalogazione dei manoscritti arabi conservati presso le biblioteche del territorio italiano". In: La presenza arabo-islamica nell'editoria italiana. Roma, 200o, Istituto poligrafico e Zecca dello Stato, p. 177-197.

7 Si vedano, oltre al già citato "I fondi di manoscritti arabi in Italia", anche le sue "Considerazioni preliminari allo studio dei manoscritti arabi”. In: Onomasticon Arabicum. Sussidi didattici. I. Roma, Istituto per l'Oriente-CNR, Institut pour l'Histoire et la Recherche des Textes-CNRs, 1975, p. 2-13; e "Il manoscritto: situazione catalografica e proposta d'una organizzazione della documentazione e delle informazioni". In: Atti del Seminario di Roma, 11-12 giugno 1980. Roma, Istituto centrale per il catalogo unico delle biblioteche italiane e per le informazioni bibliografiche, 1981, p. 38-49.

8 Traini, R. I manoscritti arabi di recente accessione della Fondazione Caetani. Catalogo di Renato Traini (Indici e sussidi bibliografici della biblioteca, 6). Roma, Accademia nazionale dei Lincei, 1967.

9 Traini, R. Catalogue of the Arabic Manuscripts in the Biblioteca Ambrosiana. Vol. IV. Nuovo Fondo: Series F-H (Nos. 1296-1778). Cinisello Balsamo (Milano), Silvana Editoriale, 2011. 
più congeniale; tra i molti risultati che produsse ${ }^{10}$ si devono ricordare almeno due fondamentali cataloghi: quello dei manoscritti arabi della Biblioteca Ambrosiana di Milano, frutto della collaborazione con Oscar Löfgren, ${ }^{11}$ e quello de I manoscritti arabi di recente accessione della Fondazione Caetani presso la Biblioteca dei Lincei. ${ }^{12}$

Non può mancare, pur nello spazio ristretto di queste pagine, un cenno ai Lincei: la biblioteca del primo piano fu per Traini un luogo particolarmente caro, "biblioteca di un prìncipe, in un palazzo di prìncipi", come scrisse anni dopo in alcune pagine in cui fortunatamente non si sottrasse a qualche cenno autobiografico che restituisce un'immagine più vicina e viva di questo studioso riservato e severo. ${ }^{13} \mathrm{Si}$ fatica un poco a immaginarlo, secondoquanto egli stesso ci racconta, nelle vesti del "nuovo, sprovveduto bibliotecario" che,appena chiamato ad assumere la cura della Sezione orientale della Biblioteca, viene condotto solennemente "attraverso la successione di sale affacciate su via della Lungara, fino a $[\ldots]$ una scrivania monumentale in legno chiaro", grato alla sorte per averlo fatto entrare in un mondo cui si affaccia "con interesse e desiderio, ma anche con il vago timore, come di una prova dall'esito niente

10 Si possono qui citare "Les manuscripts yéménites dans les bibliothèques d'Istanbul". Revue d'histoire des textes, III (1973), p. 203-230, e "I manoscritti arabi esistenti nelle biblioteche di Pavia (Collezione Robecchi Bricchetti). Atti della Accademia Nazionale dei Lincei. Rendiconti della classe di scienze morali, storiche e filologiche, Xxvin fasc. 7/12 (1974), p. 841-865. Per un elenco dettagliato della produzione, catalografica e non, di Renato Traini, si rimanda a Baffioni, "Ricordo di Renato Traini, e ad Arioli "Lessico, codici, passioni yemeniche". 35-39.

Lo studioso svedese (1898-1992) riprese e continuò il progetto di catalogazione dei manoscritti arabi della Biblioteca Ambrosiana già in parte avviato da Eugenio Griffini. A partire dal 1972, anche Traini fu chiamato a prendere parte a questo decennale lavoro, che portò alla pubblicazione del catalogo completo in 4 volumi (di cui il terzo uscito dopo la scomparsa di Löfgren e il quarto per la cura esclusiva di Renato Traini): O. Löfgren, R. Traini. Catalogue of the Arabic Manuscripts in the Biblioteca Ambrosiana. Vol. I. Antico Fondo and Medio Fondo. Vicenza, Neri Pozza Editore, 1975; Catalogue of the Arabic Manuscripts in the Biblioteca Ambrosiana. Vol. II. Nuovo Fondo: Series A-D (Nos. 1-830).Vicenza, Neri Pozza Editore, 1981; Catalogue of the Arabi Manuscripts in the Biblioteca Ambrosiana. Vol. III. Nuovo Fondo: Series E (Nos. 831-1295). Vicenza, Neri Pozza Editore, 1995 e Traini Catalogue of the Arabic Manuscripts. Vol. IV.

12 Cfr. supra n. 8.

13 Traini, R. "Leone Caetani e la sua biblioteca". In: Giornata di studio nel cinquantenario della morte di Leone Caetani. Roma, Fondazione L. Caetani dell'Accademia Nazionale dei Lincei, 1986, p. 17-37. 
affatto sicuro". Ma chiunque sia stato in questa biblioteca non farà invece fatica alcuna a immaginare (e capire) il fascino che esercitò su di lui "quella suppellettile dotta, (...) strumento e testimone delle ardue e alte ricerche, in primo luogo dello stesso Caetani, poi dei suoi collaboratori, quindi degli studiosi che avevano avuto la fortuna di poter lavorare nella sede della Fondazione. Poiché, a ben riflettere se tutti quei libri avevano, come tuttora hanno, il potere di creare attorno un'atmosfera che appunto affascina, in parte, e in parte quasi intimidisce, ciò si deve ai fantasmi di uomini illustri che essi evocano naturalmente, e ai ricordi che serbano dei successivi studiosi: tra questi e quelli, in una parola, l'aristocrazia dell'orientalismo italiano". ${ }^{14} \mathrm{E}$ tra i libri di questa gloriosa biblioteca, luogo che a lungo sarebbe stato "una parte non piccola della sua vita"15 capitava talvolta di vederlo anche dopo, durante gli anni dell'insegnamento romano o della pensione, che deve essergli giunta come l'occasione di dedicarsi ai suoi studi, finalmente libero dagli impegni dell'università.

Oltre al bel palazzo di via della Lungara, l'altro luogo che, sia pure a distanza, occupò "una parte non piccola della sua vita" è stato lo Yemen, dei cui codici Traini è stato uno dei maggiori conoscitori, studiandoli e catalogandoli per oltre trenta anni. Come è stato suggerito, ${ }^{16}$ l'interesse per lo Yemen fu probabilmente l'esito di due distinte esperienze che lo misero in contatto con questa specifica tradizione manoscritta. Infatti negli stessi anni in cui iniziò a lavorare alla catalogazione del fondo arabo dell'Ambrosiana che, grazie al fondo Caprotti, è la biblioteca europea che riunisce il maggior numero di codici yemeniti, Traini ricevette l'incarico di effettuare lo spoglio dei manoscritti zayditi dello Yemen per il progetto dell'Onomasticon Arabicum. Del resto già nel 1967, introducendo il catalogo dei Manoscritti arabi di recente accessione della Fondazione Caetani (di cui i codici yemeniti sono parte preponderante) aveva manifestato il proprio interesse per il paese sudarabico e ricordato quanto la conoscenza del patrimonio letterario del Yemen fosse ancora lacunosa. ${ }^{17}$ Una parte consistente della produzione di Traini è andata appunto a colmare

\footnotetext{
14 Ibid., p. 20.

15 Ibid., p. 19.

16 Arioli. "Ricordo di Renato Traini". 58-59.

17 Si vedano le considerazioni sulla conoscenza, nonostante tutto, scarsa del patrimonio letterario dello Yemen e sul "non disprezzabile complemento" rappresentato da 41 codici yemeniti della biblioteca della Fondazione, che giudica degni di rilievo per il contributo che possono dare allo studio della storia e delle istituzioni di questo paese. Cfr. Traini, $I$ manoscritti arabi di recente accessione. IX.
} 
tale lacuna, attraverso vari studi ${ }^{18}$ e due monografie, Sources biographiques des Zaïdites ${ }^{19}$ e Uno «specchio per principi yemenita $^{20}$ che testimoniano una conoscenza del patrimonio letterario arabo e yemenita, in particolare, difficilmente eguagliabili.

Mi pare che in questa ripetuta volontà di "colmare una lacuna" si possa trovare uno dei tratti essenziali della sua opera, e certo non sono state piccole le lacune che Renato Traini si è proposto di colmare (i vari cataloghi, il dizionario, l'esplorazione del patrimonio letterario yemenita), operazione possibile solo a uno studioso della sua pazienza e rigore metodologico. Oltre a ciò, Renato Traini ebbe anche un interesse particolare per la storia degli studi di cui si occupò ampiamente, e con la consueta erudizione, nelle pagine dedicate a Ludovico Muratori, Giorgio Levi Della Vida, Leone Caetani. ${ }^{21}$ Sono scritti in cui emerge un tono talvolta più personale, che in alcuni casi è sincero affetto e ammirazione per studiosi che considerò dei maestri (si legga la toccante orazione funebre per Francesco Gabrieli, ${ }^{22}$ o il ricordo di Oscar Löfgren), ${ }^{23} \mathrm{e}$ che in altri si traduce in uno sguardo quasi partecipe, come quello rivolto a Bernardino Baldi, autore del "primo tentativo, generoso, sebbene

18 Si vedano per esempio Traini, R. "Una storia d'amore yemenica”. OM, LIV/4 (1974), p. 173-188 e gli atti del convegno sullo Yemen da lui organizzato nell'ottobre del 2003 all'Accademia Nazionale dei Lincei, Storia e cultura dello Yemen in età islamica con particolare riferimento al periodo rasūlide (Roma, 30-31 ottobre 2003). Roma, Bardi, 2006.

19 Traini, R. Sources biographiques des Zaïdites, (années 122-120o H.). (Bibliographies, colloques, travaux préparatoires. Onomasticon Arabicum). Paris, CNRS, 1977.

Traini, R. Uno « specchio per principi » yemenita: la Nuzhat az-zurafa’ wa-tuḥfat al-hulafā’ del sultano Rasūlide al-Malik al-Afḍl (m. 778/1377). Edizione critica del testo arabo con traduzione italiana annotata. Roma, Memoria dell'Accademia Nazionale dei Lincei, ser. IX, v. XIX, fasc. 2 (2005).

Si vedano i suoi "Spunti arabistici nell'opera di L. A. Muratori”. RSo, LXXI (1997), p. 187-229; il già citato "Leone Caetani e la sua biblioteca" e "Leone Caetani islamista". In: Bonifacio VIII, i Caetani e la storia del Lazio. Atti del convegno di studi storici: Roma 3 o novembre 2000 Latina-Sermoneta, 1-2 dicembre 200o. Roma, l'Erma di Bretschneider, 2004, p. 289-306, e le pagine dedicate a Giorgio Levi Della Vida, "L'arabista". In: Giorgio Levi Della Vida nel centenario della nascita (1886-1967).Studi Semitici, n.s. IV (1988), p. 53-66.

La commemorazione che tenne nel dicembre 1998 al Dipartimento di Studi Orientali di Roma in occasione della presentazione del volume dedicato alla memoria di Francesco Gabrieli è stata pubblicata in "Francesco Gabrieli, 'uomo intero'”. OM, n.s. XVIII/3 (LXIX) (1999), p. 3-17. In precedenza aveva già ricordato il maestro nell'orazione funebre pronunciata a Santa Dorotea in Trastevere, e "In morte di Francesco Gabrieli". oM, XVI/1 (LXXVII) (1997), p. 3-4.

Traini, R. "Ricordo di Oscar Löfgren (1898-1992)". RSO, LXVII/1-2 (1993), p. 189-195. 
disordinato e incompleto, (...), di offrire comunque un panorama dello sterminato vocabolario arabo", ${ }^{24}$ parole in cui pare di sentire la vicinanza di chi ben conosceva, per esservisi misurato tre secoli dopo, la fatica di tale sterminato compito.

Nella sua produzione non manca, un poco isolata a dire il vero, anche qualche attenzione alla letteratura araba del Novecento, con la traduzione di una selezione di brani della biografia di Mihâ̄îl Nu'ayma, in un volume dedicato all'autore libanese in occasione del suo novantesimo compleanno. ${ }^{25}$ Fatta eccezione per questa incursione nella contemporaneità, Traini amò rivolgere il proprio sguardo quasi esclusivamente al mondo islamico medievale, con interessi che sono andati dalla poesia alla trasmissione del sapere a questioni lessicali, come testimonia una bibliografia in cui accanto a un nucleo consistente di scritti dedicati allo studio e alla catalogazione del patrimonio manoscritto arabo spesso, come si è detto, di contesto yemenita, ci sono comunque varie testimonianze di una curiosità intellettuale ampia che lo ha portato a spaziare dalla storia ${ }^{26}$ alla letteratura minore ${ }^{27}$ ai vari saggi di traduzione poetica (spesso in rima, con risultati di cui, lo confesso, ammiro il rigore e l'innegabile maestria ma non sempre riesco a trovare un'immediata freschezza di resa poetica, presumo irraggiungibile). ${ }^{28}$

In attesa della pubblicazione della raccolta completa dei suoi studi, ${ }^{29}$ bastino questi brevi cenni a ricordare un grande studioso forse meno noto di

24 Traini, R. “Un pioniere seicentesco degli studi arabistici e idrisiani in Italia”. In: Studi arabo-islamici in onore di Roberto Rubinacci nel suo settantesimo compleanno 2 vol. Napoli, Istituto Universitario Orientale, 1985, II, p. 699.

25 Traini, R. "Dalla autobiografia". In: A Mikhail Nu'ayma in occasione del $90^{\circ}$ compleanno. Roma, Istituto per l'Oriente, 1978, p. 105-134.

26 Si veda Traini, R. "La corrispondenza tra al-Manșūr e Muḥammad « an-Nafs az-zakiyyah »". In: Scritti in onore di Laura Veccia Vaglieri, Annali dell'Istituto Universitario Orientale, n. s. XIV (1964), p. 773-798.

27 Si veda Traini, R. "Un trattatello di galateo ed etica conviviale: le Fawāiid al-mawā’id di Ibn al-Ğazzār". In: Studi in onore di Francesco Gabrieli in onore del suo ottantesimo compleanno. 2 vol. Roma, Università di Roma "La Sapienza", Dipartimento di Studi Orientali, 1984, II, p. $783-806$.

28 Si veda per esempio il suo "Il poeta 'Amr ibn Madīkarib, Fāris al-Arab". In: Scritti in memoria di Paolo Minganti, Annali dell'Università di Cagliari, Facoltà di Scienze Politiche, IX (1983), p. 627-664.

29 Dovrebbe apparire a breve la raccolta quasi completa degli scritti di Renato Traini, per la cura di Carmela Baffioni e Paolo Nicelli, nella collana "Fonti e Studi" dell'Accademia Ambrosiana. 
quanto meriterebbe ai non arabisti, a cui, nella sua aristocratica riservatezza, forse non sarebbe spiaciuto essere ricordato con le parole di uno degli ultimi testi che tradusse:

Le nostre opere serban segno di noi, alle opere nostre dunque guardate, dopo di noi! ${ }^{30}$

30 Traini. Uno «specchio per principi $»$ yemenita. 305 . 